\title{
GENERALIZED MATRIX FUNCTIONS
}

\author{
MARVIN MARCUS $\left({ }^{1}\right)$ AND HENRYK MINC $\left({ }^{2}\right)$
}

1. Introduction. Let $S_{m}$ denote the symmetric group of degree $m$ and let $H$ be a subgroup of $S_{m}$ of order $h$. Let $\chi$ be a character of degree 1 on $H$, i.e., a nontrivial homomorphism of $H$ into the complex numbers. If $A$ is an $m$-square complex matrix we define the generalized matrix function $d_{x}$ by

$$
d_{\dot{\chi}}(A)=\sum_{\sigma \in H} \chi(\sigma) \prod_{i=1}^{m} a_{i \sigma(i)} .
$$

In [8] Schur related the function $d_{x}(A)$ to the determinant function via inequalities in the case that $A$ is a non-negative hermitian matrix. For example, one of Schur's results compares $\operatorname{det} A$ with the permanent of $A$, $\operatorname{per} A$, where

$$
\operatorname{per} A=\sum_{\sigma \in S_{m}} \prod_{i=1}^{m} a_{i \sigma(i)} \text {. }
$$

That is, per $A$ is $d_{x}(A)$ with $\chi \equiv 1$ and $H=S_{m}$.

Our results have to do with inequalities involving $d_{x}(A)$ when $A$ is a normal matrix. One of our main results is Theorem 3.1 that relates $d_{x}(A)$ to a function involving the eigenvalues of $A$. In Theorem 3.4 we also prove an extension of the known results on the van der Waerden conjecture [9] for the permanent of a doubly stochastic matrix to the $d_{x}$ functions in the case $\chi \equiv 1$. A matrix with non-negative entries is called doubly stochastic if every row and column sum is 1 , e.g., the matrix $J_{m}$ each of whose entries is $1 / \mathrm{m}$ is clearly doubly stochastic. In Corollary 3.2 we are able to extend our inequalities to arbitrary $A$ by comparing $d_{x}(A)$ with an appropriate function of the singular values of $A$. Recall that the singular values of $A$ are the non-negative square roots of the eigenvalues of $A^{*} A$.

2. Preliminary results. Let $1 \leqq m \leqq n$ and let $\Gamma_{m, n}$ denote the totality of $n^{m}$ sequences $\omega=\left(\omega_{1}, \cdots, \omega_{m}\right), 1 \leqq \omega_{i} \leqq n$. We define an equivalence relation in $\Gamma_{m, n}$ relative to $H$ : We say $\omega$ is equivalent to $\tau, \omega \sim \tau$, if and only if there exists a permutation $\sigma \in H$ such that $\omega^{\sigma}=\left(\omega_{\sigma(1)}, \cdots, \omega_{\sigma(m)}\right)=\tau$. For

\footnotetext{
Received by the editors March 23, 1964.

$\left({ }^{1}\right)$ The research of this author was supported by NSF Grant GP-1085 and the Air Force Office of Scientific Research, AFOSR-698-65.

$\left({ }^{2}\right)$ The research of this author was supported by the Air Force Office of Scientific Research Grant AFOSR-432-63.
} 
fixed $m, n$ and $H$ we denote by $\lrcorner$ a system of distinct representatives for the equivalence classes induced in $\Gamma_{m, n}$ by this equivalence relation. For example, if $H=S_{m}$ then we may take $\Delta=G_{m, n}$, where $G_{m, n}$ is the set of all $\left({ }_{m}^{n+m-1}\right)$ nondecreasing sequences $\omega, 1 \leqq \omega_{1} \leqq \cdots \leqq \omega_{m} \leqq n$. For $\gamma \in \Gamma_{m, n}$ let $\nu(\gamma)$ denote the number of permutations $\sigma \in H$ for which $\gamma^{\sigma}=\gamma$. There is a simple and important combinatorial formula that we will use repeatedly.

Lemma 2.1. Let $f: \Gamma_{m, n} \rightarrow V$ be a function on $\Gamma_{m, n}$ with values in some vector space $V$. Then

$$
\sum_{\omega \in \Gamma_{m, n}} f(\omega)=\sum_{\gamma \in\lrcorner} \frac{1}{\nu(\gamma)} \sum_{\sigma \in H} f\left(\gamma^{\sigma}\right)
$$

Proof. Since $\Delta$ is a system of distinct representatives for the equivalence induced by $H$ it is clear that $\gamma^{\sigma}$ runs over $\Gamma_{m, n}$ as $\gamma$ runs over $\Delta$ and $\sigma$ runs over $H$. Let $\gamma \in \Delta$ and let $\omega$ lie in the same equivalence class, $\bar{\gamma}$, as $\gamma$. Now suppose $\gamma^{\phi}=\omega, \phi \in H$. Then $\gamma^{\sigma}=\gamma$ if and only if $\gamma^{\sigma \phi}=\omega$. The correspondence $\sigma \phi \leftrightarrow \sigma$ is one-one and thus there are exactly $\nu(\gamma)$ permutations $\phi$ for which $\gamma^{\phi}=\omega$. Thus

$$
\begin{aligned}
\sum_{\omega \in \Gamma_{m, n}} f(\omega) & =\sum_{\gamma \in\lrcorner} \sum_{\omega \in \gamma} f(\omega) \\
& =\sum_{\gamma \in\lrcorner} \frac{1}{\nu(\gamma)} \sum_{\phi \in H} f\left(\gamma^{\phi}\right) .
\end{aligned}
$$

Let $M_{m}(V)$ be the space of $m$-multilinear functionals on a unitary space $V$ with inner product $(x, y)$. Let $V^{(m)}$ be the dual space of $M_{m}(V)$ and for $x_{i} \in V, i=1, \cdots, m$, we let $x_{1} \otimes \ldots \otimes x_{m}$ denote, as usual, the decomposable tensor satisfying $x_{1} \otimes \ldots \otimes x_{m}(f)=f\left(x_{1}, \cdots, x_{m}\right)$, for any $f \in M_{m}(V)$. The formula

$$
\left(x_{1} \otimes \ldots \otimes x_{m}, y_{1} \otimes \ldots \otimes y_{m}\right)=\prod_{i=1}^{m}\left(x_{i}, y_{i}\right)
$$

defines an inner product in $V^{(m)}$. For $\sigma \in S_{m}$ we let $P(\sigma): V^{(m)} \rightarrow V^{(m)}$ be the permutation operator which satisfies $P(\sigma) x_{1} \otimes \ldots \otimes x_{m}=x_{\sigma-1(1)} \otimes$ $\ldots \otimes x_{\sigma}-1_{(m)}$.

We then define a symmetry operator $T: V^{(m)} \rightarrow V^{(m)}$ by

$$
T=\sum_{\sigma \in H} \chi(\sigma) P(\sigma) .
$$

Clearly $T^{*}=T$ and $T^{2}=h T$. We call the subspace $T\left(V^{(m)}\right)=V_{(m)}$ the symmetry class of tensors associated with $T$. We set $T\left(x_{1} \gg \ldots x_{m}\right)$ $=x_{1} * \cdots * x_{m}$ and call this latter expression the star product of $x_{1}, \cdots, x_{m}$. for example, if $H=S_{m}, \chi(\sigma)=\operatorname{sgn} \sigma$, then the star product of $x_{1}, \cdots, x_{m}$ 
becomes the Grassman product $x_{1} \wedge \ldots \wedge x_{m}$. An important formula that follows immediately from these definitions is

$$
\left(x_{1} * \ldots * x_{m}, y_{1} * \ldots * y_{m}\right)=h \sum_{\sigma \in H} \chi(\sigma) \prod_{i=1}^{m}\left(x_{i}, y_{\sigma(i)}\right)
$$

At this point we note the connection between the generalized matrix function $d_{x}$ defined in $\$ 1$ and the formula (2.3): if $A=\left(\left(x_{i}, x_{j}\right)\right), i, j=1, \cdots, m$, then

$$
\left(x_{1} * \ldots * x_{m}, y_{1} * \ldots * y_{m}\right)=h d_{x}(A) \text {. }
$$

If $e_{1}, \cdots, e_{n}$ is a basis for $V$ then it is easy to prove that the tensors $e_{\gamma}=e_{\gamma_{1}} * \cdots * e_{\gamma_{m}}, \quad \gamma \in \Delta$, span $V_{(m)}$. Let $\gamma \in \Delta$ and consider the sum $\sum_{\gamma^{\sigma}=\gamma} \chi(\sigma)$ where the summation extends over all $\sigma \in H$ for which $\gamma^{\sigma}=\gamma$. Clearly this set is a subgroup of $H$, call it $H_{\gamma}$, and hence $\sum_{\gamma^{\sigma}=\gamma} \chi(\sigma)$ is 0 or the order of $H_{\gamma}$. But $\nu(\gamma)$ is by definition the order of $H_{\gamma}$. We let $\bar{\Delta}$ be the subset of $\Delta$ consisting of those $\gamma \in \Delta$ for which $\sum_{\gamma^{\sigma}=\gamma} \chi(\sigma)=\nu(\gamma)$.

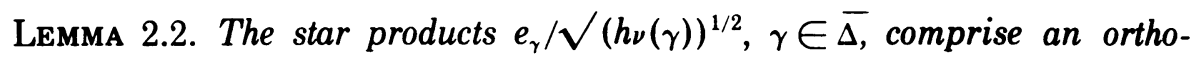
normal basis for $V_{(m)}$ when $e_{1}, \cdots, e_{n}$ is an orthonormal basis of $V$.

Proof. Let $\gamma$ and $\tau$ be in $\Delta$. Compute from (2.3) that

$$
\begin{aligned}
\left(e_{\gamma}, e_{\tau}\right) & =h \sum_{\sigma \in H} \chi(\sigma) \prod_{i=1}^{m}\left(e_{\gamma_{i}}, e_{\tau_{\sigma}(i)}\right) \\
& =h \sum_{\sigma \in H} \chi(\sigma) \delta_{\gamma, \tau} \sigma .
\end{aligned}
$$

Since both $\gamma$ and $\tau$ are members of a system of distinct representatives it follows that $\tau^{\circ}=\gamma$ if and only if $\tau=\gamma$. Thus

$$
\left(e_{\gamma}, e_{r}\right)=h \delta_{\gamma, r} \sum_{\sigma \in H} \chi(\sigma) \delta_{\gamma, \gamma} \sigma=h \delta_{\gamma, r} \sum_{\gamma^{\sigma} \approx \gamma} \chi(\sigma) .
$$

Now $\sum_{\gamma^{\sigma}=\gamma} \chi(\sigma)$ is 0 or $\nu(\gamma)$ according as $\gamma \notin \bar{\Delta}$ or $\gamma \in \bar{\Delta}$. Thus $e_{\gamma}=0$ if $\gamma \notin \bar{\Delta}$ and $\left\|e_{\gamma}\right\|^{2}=h_{\nu}(\gamma)$ if $\gamma \in \bar{\Delta}$, completing the proof.

If $A$ is $n$-square and $\omega, \tau \in \Gamma_{m, n}$ then $A[\omega \mid \tau]$ denotes the $m$-square submatrix whose $(i, j)$ entry is $a_{\omega_{i}, r_{j}}$.

Lemma 2.3 (Generalized Cauchy-Binet Theorem). If $A$ is $m \times n$ and $B$ is $n \times m$ then

$$
d_{\chi}(A B)=\sum_{\gamma \in \mathcal{J}} \frac{1}{\nu(\gamma)} d_{\chi}(A[1, \cdots, m \mid \gamma]) d_{\chi}(B[\gamma \mid 1, \cdots, m]) .
$$

Proof. Let $e_{1}, \cdots, e_{n}$ be an orthonormal basis of $V$ and let 


$$
x_{i}=\sum_{s=1}^{n} a_{i s} e_{s}, \quad y_{j}=\sum_{t=1}^{n} \bar{b}_{t j} e_{t}, \quad i, j=1, \cdots, m .
$$

Then

$$
\begin{aligned}
\left(x_{i}, y_{j}\right) & =\sum_{k=1} a_{i k} b_{k j}=(A B)_{i j}, \\
\left(x_{i}, e_{\gamma_{j}}\right) & =a_{i \gamma_{j}},\left(e_{\gamma_{i}}, y_{j}\right)=b_{\gamma_{i}},
\end{aligned}
$$

and hence

$$
\left(\left(x_{i}, e_{\gamma_{j}}\right)\right)=A[1, \cdots, m \mid \gamma]
$$

and

$$
\left(\left(e_{\gamma_{i}}, y_{j}\right)\right)=B[\gamma \mid 1, \cdots, m] .
$$

By Parseval's equality applied in $V_{(m)}$ to the orthonormal basis of star products $\mathrm{e}_{\gamma} /\left(h_{\nu}(\gamma)\right)^{1 / 2}, \gamma \in \bar{\Delta}$, we compute

$$
\begin{aligned}
d_{\chi}(A B) & =\frac{1}{h}\left(x_{1} * \ldots * x_{m}, y_{1} * \cdots * y_{m}\right) \\
& =\frac{1}{h} \sum_{\gamma \in J}\left(x_{1} * \ldots * x_{m}, \frac{e_{\gamma}}{(h \nu(\gamma))^{1 / 2}}\right)\left(\frac{e_{\gamma}}{\left(h_{\nu}(\gamma)\right)^{1 / 2}}, y_{1} * \cdots * y_{m}\right) \\
& =\frac{1}{h} \sum_{\gamma \in J} \frac{1}{h \nu(\gamma)} h d_{\chi}(A[1, \cdots, m \mid \gamma]) h d_{\chi}(B[\gamma \mid 1, \cdots, m]) \\
& \left.=\sum_{\gamma \in\lrcorner} \frac{1}{\nu(\gamma)} d_{\chi}(A[1, \cdots, m \mid \gamma]) d_{\chi}(B|\gamma| 1, \cdots, m]\right) .
\end{aligned}
$$

We remark that since $e_{\gamma}=0$ for $\gamma \notin \bar{\Delta}$, we can replace $\bar{\Delta}$ by $\Delta$ in (2.5). This trivial observation, when translated into matrix language, has a rather startling corollary: if $\gamma \in \Gamma_{m, n}$ is such that $\sum_{\gamma^{\sigma}=\gamma, \sigma \in H} \chi(\sigma)=0$ then for any $m \times n$ matrix $A$ we have

$$
d_{\chi}(A[1, \cdots, m \mid \gamma])=0 .
$$

We apply Lemma 2.3 to obtain an important result involving values of $d_{x}$ on normal matrices. Let $m_{t}(\gamma)$ denote the number of occurrences of $t$ in the sequence $\gamma \in \Gamma_{m, n}$, e.g., $m_{4}((1,2,2,4,4))=2$.

Theorem 2.1. If $A=U^{*} D U$, where $U$ is $m$-square unitary and $D$ $=\operatorname{diag}\left(r_{1}, \cdots, r_{m}\right)$, then

$$
d_{\chi}(A)=\sum_{\gamma \in \mathcal{J}} \frac{1}{\nu(\sigma)}\left|d_{\chi}(U[\gamma \mid 1, \cdots, m])\right|^{2} \prod_{t=1}^{m} r_{t}^{m_{t}(\gamma)} .
$$


Proof. From Lemma 2.3 we compute that

$$
d_{x}(A)=\sum_{\gamma \in \bar{\Delta}} \frac{1}{\nu(\gamma)} d_{x}\left(U^{*}[1, \cdots, m \mid \gamma]\right) d_{x}((D U)[\gamma \mid 1, \cdots, m]) .
$$

Moreover,

$$
\begin{aligned}
d_{x}\left(U^{*}[1, \cdots, m \mid \gamma]\right) & =\overline{\sum_{\sigma \in H} \chi(\sigma) \prod_{i=1}^{m} \bar{u}_{\gamma_{\sigma}(i), i}} \\
& =\overline{\sum_{\sigma \in H} \overline{\chi(\sigma)} \prod_{i=1}^{m} u_{\gamma_{\sigma(i), i}}} \\
& =\overline{\left.\sum_{\phi \in H} \overline{\chi\left(\phi^{-1}\right.}\right) \prod_{i=1}^{m} u_{\gamma_{i}, \phi(i)}} \\
& =\overline{\sum_{\phi \in H} \chi(\phi) \prod_{i=1}^{m} u_{\gamma_{i}, \phi(i)}} \\
& =\overline{d_{\chi}(U[\gamma \mid 1, \cdots, m]) .}
\end{aligned}
$$

Also

$$
d_{\chi}((D U)[\gamma \mid 1, \cdots, m])=\prod_{t=1}^{m} r_{t}^{m_{t}(\gamma)} d_{x}(U[\gamma \mid 1, \cdots, m])
$$

and (2.6) follows.

We can use Theorem 2.1 to derive special relations that must obtain among the values of $d_{x}$ on certain matrices constructed from the rows of a unitary matrix. This will be used subsequently to yield an upper bound for $\left|d_{x}(A)\right|$ when $A$ is normal.

TheOREM 2.2. If $U$ is $m$-square unitary and $1 \leqq p \leqq m$, then

$$
\sum_{\gamma \in \bar{J}} \frac{m_{p}(\gamma)}{\nu(\gamma)}\left|d_{x}(U[\gamma \mid 1, \cdots, m])\right|^{2}=1 .
$$

Proof. In formula (2.6) we regard $r_{1}, \cdots, r_{m}$ as variables and $U$ as a fixed matrix. We then differentiate both sides of (2.6) with respect to $r_{p}$ and evaluate both sides of the resulting equation at $\left(r_{1}, \cdots, r_{m}\right)=(1, \cdots, 1)$ $=e$. First observe that

$$
d_{\chi}(A)=a_{s t} \sum_{\sigma(s)=t} \chi(\sigma) \prod_{i=1, i \neq s}^{m} a_{i \sigma(i)}+\sum^{\prime},
$$


where the first summation extends over all $\sigma \in H$ for which $\sigma(s)=t$ (if any) and $\sum^{\prime}$ does not involve $a_{s t}$. Thus

$$
\frac{\partial d_{\chi}(A)}{\partial a_{s t}}=\sum_{\sigma(s)=t} \chi(\sigma) \prod_{i=1, i \neq s}^{m} a_{i \sigma(i)}
$$

Since $d_{x}(A)$ can be regarded as a composite function of $r_{1}, \cdots, r_{m}$ we have

$$
\frac{\partial d_{\chi}(A)}{\partial r_{p}}=\sum_{s, t=1}^{m} \frac{\partial d_{\chi}(A)}{\partial a_{s t}} \frac{\partial a_{s t}}{\partial r_{p}}
$$

We evaluate $(2.8)$ at $e=(1, \cdots, 1)$. Since $A$ collapses to $I_{m}$ for $r_{1}=\ldots=r_{m}$ $=1, a_{i \sigma(i)}=\delta_{i \sigma(i)}, i=1, \cdots, m$, and hence

$$
\left.\frac{\partial d_{x}(A)}{\partial a_{s t}}\right|_{e}=0
$$

if $s \neq t$. If $s=t$ then

$$
\begin{aligned}
\left.\frac{\partial d_{\chi}(A)}{\partial a_{s s}}\right|_{e} & =\sum_{\sigma(s)=s} \chi(\sigma) \prod_{i=1, \mathrm{i} \neq s}^{m} \delta_{i \sigma(i)} \\
& =1
\end{aligned}
$$

Thus we compute from (2.9) that

$$
\begin{aligned}
\left.\frac{\partial d_{\chi}(A)}{\partial r_{p}}\right|_{e} & =\left.\sum_{s=1}^{m} \frac{\partial a_{s s}}{\partial r_{p}}\right|_{e}=\left.\frac{\partial}{\partial r_{p}}\left(\sum_{s=1}^{m} a_{s s}\right)\right|_{e} \\
& =\left.\frac{\partial}{\partial r_{p}}(\operatorname{tr}(A))\right|_{e}=\left.\frac{\partial}{\partial r_{p}}\left(\sum_{s=1}^{m} r_{s}\right)\right|_{e}=1 .
\end{aligned}
$$

If we differentiate the right side of (2.6) with respect to $r_{p}$ we obtain

$$
\frac{\partial d_{\chi}(A)}{\partial r_{p}}=\sum_{\gamma \in \mathcal{J}} \frac{1}{\nu(\gamma)}\left|d_{\chi}(U[\gamma \mid 1, \cdots, m])\right|^{2} m_{p}(\gamma) r_{p}^{m_{p}(\gamma)-1} \prod_{t=1, t \neq p}^{m} r_{t}^{m_{t}(\gamma)}
$$

Thus

$$
\left.\frac{\partial d_{\chi}(A)}{\partial r_{p}}\right|_{e}=\sum_{\gamma \in \mathcal{J}} \frac{m_{p}(\gamma)}{\nu(\gamma)}\left|d_{\chi}(U[\gamma \mid 1, \cdots, m])\right|^{2},
$$

completing the proof.

Our last preliminary topic concerns the analysis of equality between star products. This investigation is important in order to decide the cases of equality in certain inequalities in $\$ 3$. 
It is proved in [6] that for $H=S_{m}$ and $\chi \equiv 1, x_{1} * \ldots * x_{m}=0$ if and only if some $x_{i}=0$. Also $x_{1} * \cdots * x_{m}=y_{1} * \cdots * y_{m} \neq 0$ if and only if $x_{i}$ $=d_{i} y_{\sigma(i)}, i=1, \cdots, m$, for some $\sigma \in S_{m}$ and scalars $d_{i}, \prod_{i=1}^{m} d_{i}=1$.

LEMmA 2.4. If $x_{i}$ and $y_{i}$ are in $V, i=1, \cdots, m, H$ is any subgroup, and $x \equiv 1$, then

(i) $x_{1} * \cdots * x_{m}=0$ if and only if some $x_{i}=0$;

(ii) if $x_{1} * \ldots * x_{m}=y_{1} * \cdots * y_{m} \neq 0$ then there exists $a \sigma \in S_{m}$ and constants $d_{i} \neq 0, i=1, \cdots, m$, such that $x_{i}=d_{i} y_{o(i)}, i=1, \cdots, m$. Moreover, $\prod_{i=1}^{m} d_{i}=1$.

Proof. Let $R=\sum_{\sigma \in S_{m}} P(\sigma)$. Observe that if $T=\sum_{\sigma \in H} P(\sigma)$ then

$$
T R=R T=h R .
$$

Thus if $x_{1} * \ldots * x_{m}=0$ then $T\left(x_{1} \otimes \cdots \otimes x_{m}\right)=0$ and hence $R T\left(x_{1} \otimes \cdots \otimes x_{m}\right)$ $=0$. From (2.3) we see that

$$
\begin{aligned}
0 & =\left\|R T x_{1} \otimes \ldots \otimes x_{m}\right\|^{2}=h^{2}\left\|R x_{1} \otimes \cdots \otimes x_{m}\right\|^{2} \\
& =h^{2} m ! \operatorname{per}\left(\left(x_{i}, x_{j}\right)\right) .
\end{aligned}
$$

It is proved in [1] that

$$
\operatorname{per}\left(\left(x_{i}, x_{j}\right)\right) \geqq \prod_{i=1}^{m}\left(x_{i}, x_{i}\right)=\prod_{i=1}^{m}\left\|x_{i}\right\|^{2} .
$$

Hence $\prod_{i=1}^{m}\left\|x_{i}\right\|^{2}=0$ and thus some $x_{i}=0$. This proves (i). Similarly, if $T\left(x_{1} \otimes \cdots \otimes x_{m}\right)=T\left(y_{1} \otimes \ldots \otimes y_{m}\right)$ then $R\left(x_{1} \otimes \cdots \otimes x_{m}\right)=R\left(y_{1} \otimes \cdots \otimes y_{m}\right)$ and clearly if some $x_{i}$ or $y_{j}$ were 0 then $x_{1} * \cdots * x_{m}=y_{1} * \cdots * y_{m}=0$. Hence no $x_{i}$ or $y_{j}$ is 0 and we can use [6, Theorem 3] to conclude (ii). We remark that it is an open question at present whether or not the permutation $\sigma$ in (ii) above can be chosen to be in the subgroup $H$.

3. Main results. We first state and prove an inequality for the values of the $d_{x}$ function on normal matrices.

THEOREM 3.1. If $A$ is $m$-square normal with eigenvalues $r_{1}, \cdots, r_{m}$ then

$$
\left|d_{x}(A)\right| \leqq \frac{1}{m} \sum_{t=1}^{m}\left|r_{t}\right|^{m}
$$

Proof. For $\gamma \in \bar{\Delta}$ let

$$
c_{\gamma}=\left|d_{x}(U[\gamma \mid 1, \cdots, m])\right|^{2},
$$

where

$$
A=U^{*} \operatorname{diag}\left(r_{1}, \cdots, r_{m}\right) U
$$

and $U$ is $m$-square unitary. Then, from (2.6), 


$$
\begin{aligned}
\left|d_{x}(A)\right| & =\left|\sum_{\gamma \in \bar{\Delta}} \frac{c_{\gamma}}{\nu(\gamma)} \prod_{t=1}^{m} r_{t}^{m_{t}(\gamma)}\right| \\
& \leqq \sum_{\gamma \in \bar{J}^{-}} \frac{c_{\gamma}}{\nu(\gamma)} \prod_{t=1}^{m}\left|r_{t}\right|^{m_{t}(\gamma)} \\
& \leqq \sum_{\gamma \in \bar{J}^{-}} \frac{c_{\gamma}}{\nu(\gamma)}\left(\frac{1}{m} \sum_{t=1}^{m} m_{t}(\gamma)\left|r_{t}\right|\right)^{m} \\
& \leqq \sum_{\gamma \in \bar{\Delta}^{-}} \frac{c_{\gamma}}{\nu(\gamma)} \frac{1}{m} \sum_{t=1}^{m} m_{t}(\gamma)\left|r_{t}\right|^{m} \\
& =\frac{1}{m} \sum_{t=1}^{m}\left|r_{t}\right|^{m} \sum_{\gamma \in \bar{J}} \frac{m_{t}(\gamma) c_{\gamma}}{\nu(\gamma)} \\
& =\frac{1}{m} \sum_{t=1}^{m}\left|r_{t}\right|^{m} .
\end{aligned}
$$

The last equality follows from Theorem 2.2.

Applying the Cauchy-Schwarz inequality to (2.4) yields

Theorem 3.2. If $A$ is $m \times n$ and $B$ is $n \times m$ then

$$
\left|d_{x}(A B)\right|^{2} \leqq d_{x}\left(A A^{*}\right) d_{x}\left(B^{*} B\right) \text {. }
$$

In case $\chi \equiv 1$, equality holds in (3.2) only if (i) $A$ has a zero row, or (ii) $B$ has zero column or (iii) $A=D P B^{*}$, where $D$ is a diagonal matrix, and $P$ is a permutation matrix.

The cases of equality follow from Lemma 2.4 .

Theorems 3.1 and 3.2 yield the following corollaries.

CoRollary 3.1. If $N$ is $m$-square normal with eigenvalues $r_{1}, \cdots, r_{m}$ then

$$
|\operatorname{per} N| \leqq \frac{1}{m} \sum_{i=1}^{m}\left|r_{i}\right|^{m}
$$

If in addition $N$ is doubly stochastic then

$$
|\operatorname{per} N| \leqq \frac{\rho(N)}{m}
$$

where $\rho(N)$ denotes the rank of $N$. The inequality (3.4) is strict unless either $N$ is a permutation matrix or $m=2$ and $N=J_{2}$.

Proof. The inequality (3.3) follows immediately from Theorem 3.1. The inequality (3.4) including the discussion of equality is found in [4]. 
COROLlary 3.2. If $A$ is an arbitrary complex matrix with singular values $\alpha_{1}, \cdots, \alpha_{m}$ then

$$
\left|d_{x}(A)\right|^{2} \leqq \frac{1}{m} \sum_{i=1}^{m} \alpha_{i}^{2 m}
$$

Equality holds for $\chi \equiv 1$ in (3.5) if and only if $A=\alpha \operatorname{diag}\left(e^{i \theta_{1}}, \ldots, e^{i \theta_{m}}\right) P$, where $P$ is a permutation matrix corresponding to some permutation $\sigma$ in the group $H$.

Proof. We use Theorem 3.2 and Theorem 3.1 applied to the hermitian matrix $A A^{*}$ to conclude

$$
\left|d_{\mathrm{x}}(A)\right|^{2} \leqq d_{\mathrm{x}}\left(A A^{*}\right) \leqq \frac{1}{m} \sum_{i=1}^{m} \alpha_{i}^{2 m} .
$$

If equality holds for $\chi \equiv 1$ in (3.6) then (i) $A$ has a zero row or column or (ii) $A$ has no zero row or column and $A=D P$, where $D$ is a diagonal matrix and $P$ is a permutation matrix. Suppose (i); then $d_{x}(A)=0$ $=(1 / m) \sum_{i=1}^{m} \alpha_{i}^{2 m}$ and hence $\alpha_{1}=\cdots=\alpha_{m}=0$. It follows that $A=0$. If (ii), then $D=\operatorname{diag}\left(d_{1}, \cdots, d_{m}\right)$ has no zero main diagonal elements. Thus

$$
\begin{aligned}
d_{x}\left(A A^{*}\right) & =d_{\times}\left(D D^{*}\right)=\prod_{i=1}^{m}\left|d_{i}\right|^{2} \\
& =\frac{1}{m} \sum_{i=1}^{m} \alpha_{i}^{2 m}=\frac{1}{m} \sum_{i=1}^{m}\left|d_{i}\right|^{2 m}
\end{aligned}
$$

Hence

$$
\left|d_{1}\right|=\cdots=\left|d_{m}\right|=\alpha
$$

Set $d_{j}=\alpha e^{i \theta_{j}}$ and then

$$
A=\alpha \operatorname{diag}\left(e^{i \theta_{1}}, \cdots, e^{i \theta_{m}}\right) P .
$$

Now,

$$
d_{x}(A)= \begin{cases}\chi(\sigma) \alpha^{m} e^{i \theta} & \text { if } \sigma \in H, \\ 0 & \text { if } \sigma \notin H .\end{cases}
$$

If $\sigma \notin H$ then $0=d_{x}(A)$ and once again we would conclude $A=0$.

CoRollary 3.3. If $A$ is an arbitrary $m$-square doubly stochastic matrix then

$$
\text { per } A \leqq\left(\frac{\rho(A)}{m}\right)^{1 / 2} \text {. }
$$


Equality holds in (3.7) if and only if $\rho(A)=m$ and $A$ is a permutation matrix.

Proof. By Theorem 3.2 and Corollary 3.1 applied to the permanent function we have

$$
(\operatorname{per} A)^{2} \leqq \operatorname{per} A A^{*} \leqq \frac{\rho\left(A A^{*}\right)}{m}=\frac{\rho(A)}{m}
$$

and (3.7) follows. If equality holds then either $A A^{*}$ is a permutation matrix or $m=2$ and $A A^{*}=J_{2}$. If $A A^{*}=J_{2}$ then $\rho(A)=1$ and since $A$ is doubly stochastic, $A$ would be $J_{2}$. But $\left(\operatorname{per} J_{2}\right)^{2}=1 / 4$ while $\rho(A) / 2=1 / 2$. If $A A^{*}=P$, where $P$ is a permutation matrix then both $A$ and $A^{-1}$ have non-negative entries and thus $A$ is a permutation matrix.

We next obtain an inequality relating the eigenvalues of an $n$-square matrix $A$ with the values of $d_{x}$ on a principal submatrix of $A$.

Theorem 3.3. If $A$ is an $n$-square positive semi-definite hermitian matrix with eigenvalues $r_{1} \geqq \cdots \geqq r_{n}$ and $B$ is an $m$-square principal submatrix of $A, 1 \leqq m \leqq n$, then

$$
\prod_{j=1}^{m} r_{n-j+1} \leqq d_{\chi}(B) \leqq \frac{1}{m} \sum_{j=1}^{m} r_{j}^{m}
$$

Proof. Let $(x, y)$ denote the usual inner product in the space of $n$-tuples. Since $B$ is a principal submatrix of $A$ there exists an orthonormal set of vectors $e_{1}, \cdots, e_{m}$ such that $b_{i j}=\left(A e_{j}, e_{i}\right)$. Then by $[4$, Corollary 1]

$$
\begin{aligned}
d_{x}(B) & \geqq \operatorname{det} B \\
& =\operatorname{det}\left(\left(A e_{j}, e_{i}\right)\right) \\
& =\operatorname{det}\left(\left(A e_{i}, e_{j}\right)\right) \\
& =\left(C_{m}(A) e_{1} \wedge \cdots \wedge e_{m}, e_{1} \wedge \cdots \wedge e_{m}\right) \\
& \geqq \prod_{j=1}^{m} r_{n-j+1} .
\end{aligned}
$$

The latter inequality is found in [3] where the general extremal problem for Grassmann compounds $C_{m}(A)$ is analyzed.

To prove the other inequality in (3.8) we use Theorem 3.1 to obtain

$$
d_{\chi}(B) \leqq \frac{1}{m} \sum_{i=1}^{m} s_{i}^{m}
$$

where $s_{1} \geqq \cdots \geqq s_{m}$ are the eigenvalues of $B$. By the Cauchy inequalities (see [5, Chapter II, 4.4.7]), $s_{i} \leqq r_{i}, i=1, \cdots, m$, and the result follows. In [6] it was proved that per $A \geqq m ! / m^{m}$ when $A$ is an $m$-square positive 
semi-definite hermitian doubly stochastic matrix. Later in [7] this result was extended to a slightly larger class of matrices. We now extend the result to a more general class of matrices and to the generalized matrix functions.

THEOREM 3.4. Let $A$ be an $m$-square non-negative hermitian matrix whose ith row sum is $s_{i}, i=1, \cdots, m$. Assume that $\sum_{i=1}^{m} s_{i}=s \neq 0$. If $\chi \equiv 1$

$$
d_{x}(A) \geqq \frac{h}{s^{m}} \prod_{i=1}^{m}\left|s_{i}\right|^{2} .
$$

Equality holds in (3.9) if and only if either (i) a row of $A$ is zero or (ii) $\rho(A)=1$.

Proof. Since $A$ is positive semi-definite hermitian it is a Gram matrix based on some set of vectors $x_{1}, \cdots, x_{m}$, i.e., $a_{i j}=\left(x_{i}, x_{j}\right)$. From (2.4) we have

$$
\left\|x_{1} * \cdots * x_{m}\right\|^{2}=h d_{x}(A) \text {. }
$$

Let $u=x_{1}+\cdots+x_{m}$ and then compute immediately that $\|u\|^{2}=s$. We assumed $s \neq 0$ and hence it follows that $s>0$ and $u \neq 0$. Let $v=u * \ldots * u$ and from Lemma 2.4 observe that $v \neq 0$. Thus from (3.10), and the CauchySchwarz inequality

$$
\begin{aligned}
h d_{x}(A) & \geqq \frac{1}{\|v\|^{2}}\left|\left(x_{1} * \ldots * x_{m}, u * \ldots * u\right)\right|^{2} \\
& =\frac{1}{\|v\|^{2}}\left|h d_{x}\left(\left(x_{i}, u\right)\right)\right|^{2} .
\end{aligned}
$$

Note that $\left(x_{i}, u\right)=s_{i}$ and thus

$$
\begin{aligned}
h d_{\chi}(A) & \geqq \frac{h^{2}}{\|v\|^{2}}\left|h \prod_{i=1}^{m} s_{i}\right|^{2} \\
& =\frac{h^{4}}{\|v\|^{2}} \prod_{i=1}^{m}\left|s_{i}\right|^{2}
\end{aligned}
$$

Now,

$$
\begin{aligned}
\|v\|^{2} & =\|u * \cdots * u\|^{2} \\
& =h d_{\chi}((u, u)) \\
& =h d_{x}((s)) \\
& =h^{2} s^{m} .
\end{aligned}
$$

Combining (3.11) and (3.12) we have the inequality (3.9). 
For equality to hold in (3.9) it is clear from the cases of equality in the Cauchy-Schwarz inequality that $x_{1} * \ldots * x_{m}$ and $u * \cdots * u$ must be linearly dependent. Since $u \neq 0$ we have, by Lemma 2.4 , either (i) $x_{i}=0$ for some $i$, or (ii) $x_{i}=d_{i} u, d_{i} \neq 0, i=1, \cdots, m$, i.e., $\rho(A)=1$. If (i), then clearly $A$ has a zero row. Conversely, if $A$ has a zero row then obviously both sides of (3.9) are 0 . If $\rho(A)=1$ so that

$$
a_{i j}=d_{i} \bar{d}_{j}, \quad i, j=1, \cdots, m,
$$

then

$$
d_{\chi}(A)=h \prod_{i=1}^{m}\left|d_{i}\right|^{2}
$$

whereas,

$$
s_{i}=d_{i} \sum_{j=1}^{m} \bar{d}_{j}
$$

Then

$$
\prod_{i=1}^{m}\left|s_{i}\right|^{2}=\prod_{i=1}^{m}\left|d_{i}\right|^{2}\left|\sum_{j=1}^{m} d_{j}\right|^{2 m}
$$

and

$$
s^{m}=\left(\sum_{i=1}^{m} s_{i}\right)^{m}=\left(\sum_{i=1}^{m} d_{i} \sum_{j=1}^{m} \bar{d}_{j}\right)^{m}=\left|\sum_{i=1}^{m} d_{i}\right|^{2 m}
$$

Hence

$$
\frac{h}{s^{m}} \prod_{i=1}^{m}\left|s_{i}\right|^{2}=h \prod_{i=1}^{m}\left|d_{i}\right|^{2}=d_{x}(A),
$$

completing the proof.

Corollary 3.4. If $A$ is an m-square doubly stochastic positive semidefinite hermitian matrix, then

$$
\text { per } A \geqq \frac{m !}{m^{m}} .
$$

We re-examine Theorem 2.1 in anticipation of applying it to special choices of $H$. First observe that each $\gamma(t)=(t, \cdots, t), t=1, \cdots, m$, is in $\Delta$ because it is the sole member of an equivalence class induced in $\Gamma_{m, n}$ by $H$. We assume here that $\chi \equiv 1$ so that

$$
\sum_{\sigma \in H, \gamma^{\sigma}(t)=\gamma(t)} \chi(\sigma)=\nu(\gamma(t))=h, \quad \text { i.e., } \gamma(t) \in \bar{\Delta}, t=1, \cdots, m .
$$


If the eigenvalues $r_{1}, \cdots, r_{m}$ of $A$ are non-negative, then from (2.6) we can conclude that if $\theta$ is any subset of $\bar{\Delta}$ then

$$
d_{\mathrm{x}}(A) \geqq \sum_{\gamma \in \theta} \frac{1}{\nu(\gamma)}\left|d_{\mathrm{x}}(U[\gamma \mid 1, \cdots, m])\right|^{2} \prod_{t=1}^{m} r_{t}^{m_{t}(\gamma)} .
$$

If $\theta$ is chosen to be the set of $\gamma(t), t=1, \cdots, m$, we obtain

$$
\begin{aligned}
d_{x}(A) & \geqq \sum_{t=1}^{m} \frac{1}{h}\left|d_{x}(U[t, \cdots, t \mid 1, \cdots, m])\right|^{2} r_{t}^{m} \\
& =\frac{1}{h} \sum_{t=1}^{m}\left|h \prod_{j=1}^{m} u_{t j}\right|^{2} r_{t}^{m} \\
& =h \sum_{t=1}^{m}\left|\prod_{j=1}^{m} u_{t j}\right|^{2} r_{t}^{m} .
\end{aligned}
$$

The eigenvector of $A$ corresponding to $r_{t}$ is just $\left(\bar{u}_{t 1}, \cdots, \bar{u}_{t m}\right)$.

THEOREM 3.5. If $\chi \equiv 1$ and $A$ is positive semi-definite hermitian and $\xi_{t}$ is the product of the squares of the absolute values of the coordinates of the unit eigenvector corresponding to $r_{t}$ then

$$
d_{\mathrm{x}}(A) \geqq h \sum_{t=1}^{m} \xi_{t} r_{t}^{m}
$$

For example, if $\left(1 /(m)^{1 / 2}, \cdots, 1 /(m)^{1 / 2}\right)$ is an eigenvector of $A$ corresponding to $r$ then

$$
d_{\chi}(A) \geqq \frac{h r^{m}}{m^{m}} .
$$

We remark that in case $A$ is doubly stochastic and $H=S_{m}$ we have from (3.15)

$$
\text { per } A \geqq \frac{m !}{m^{m}},
$$

which is once again the Corollary 3.4.

Let $A$ be an $m$-square circulant based on the first row $\left(c_{0}, c_{m-1}, \cdots, c_{1}\right)$. If $\psi(\lambda)$ is the polynomial $\sum_{t=0}^{m-1} c_{t} \lambda^{t}$ and $\epsilon=e^{i 2 x / m}$ then the eigenvalues of $A$ are $r_{p}=\psi\left(\epsilon^{p}\right)$ with corresponding eigenvectors

$$
v_{p}=\frac{1}{(m)^{1 / 2}}\left(\epsilon^{m-p}, \epsilon^{2(m-p)}, \cdots, \epsilon^{m(m-p)}\right), \quad p=1, \cdots, m .
$$

The values of $\xi_{p}$ are thus $1 / m^{m}, p=1, \cdots, m$. 
If in (3.13) we allow $\theta$ to be the set $\gamma(t), t=1, \cdots, m$, together with the sequence $(1,2, \cdots, m)$ we conclude immediately that

$$
d_{\chi}(A) \geqq \frac{h}{m^{m}} \sum_{i=1}^{m} r_{i}^{m}+\frac{1}{m^{m}}\left|d_{x}(R)\right|^{2} \operatorname{det} A,
$$

where $R$ is the $m$-square matrix whose $(s, t)$ entry is $\epsilon^{s t}$.

Thus we have

Corollary 3.5. If $A$ is a positive semi-definite $m$-square hermitian circulant, $\chi \equiv 1$ and $\epsilon=e^{i 2 \pi / m}$, then

$$
d_{\chi}(A) \geqq \frac{1}{m^{m}}\left[h \cdot \operatorname{tr}\left(A^{m}\right)+\operatorname{det} A\left|\sum_{\sigma \in H}^{m} \prod_{s=1}^{m} \epsilon^{s \sigma(s)}\right|^{2}\right]
$$

\section{REFERENCES}

1. Marvin Marcus, The permanent analogue of the Hadamard determinant theorem, Bull. Amer. Math. Soc. 69 (1963), 494-496.

2. in Matrix Theorem (Proc. Advanced Seminar, Math. Res. Center, U. S. Army, Univ. of Wisconsin, Madison, Wis., 1963), pp. 61-80, Univ. Wisconsin Press, Madison, Wis., 1964.

3. M. Marcus and J. L. McGregor, Extremal properties of Hermitian matrices, Canad. J. Math. 8 (1956), 524-531.

4. Marvin Marcus and Henryk Minc, Inequalities for general matrix functions, Bull. Amer. Math. Soc. 70 (1964), 308-313.

5. __ A survey of matrix theory and matrix inequalities, Allyn and Bacon, Boston, Mass., 1964.

6. Marvin Marcus and Morris Newman, Inequalities for the permanent function, Ann. of Math. (2) 75 (1962), 47-62.

7. Henryk Minc, $A$ note on an inequality of $M$. Marcus and $M$. Newman, Proc. Amer. Math. Soc. 14 (1963), 890-892.

8. I. Schur, Uber endliche Gruppen und Hermitesche Formen, Math. Z. 1 (1918), 184-207.

9. B. L: van der Waerden, Aufgabe 45, Jber. Deutsch. Math.-Verein. 35 (1926), 117.

\section{UNIVERSITY OF CALIFORNIA,}

Santa Barbara, California 